
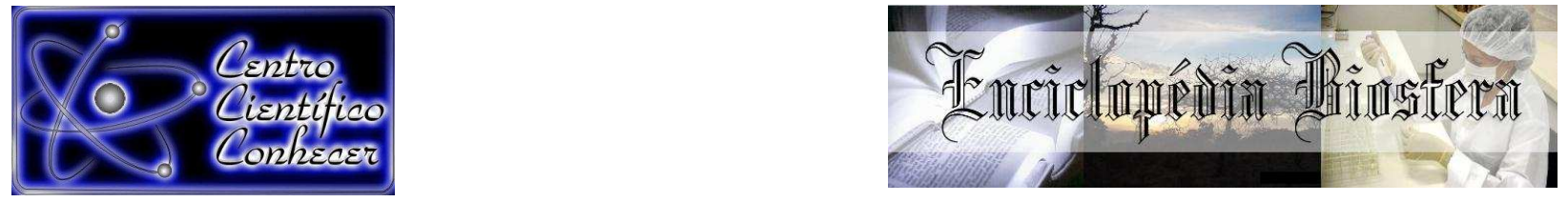

\title{
ATRATIVIDADE DE Cosmopolites sordidus POR GENÓTIPOS DE BANANEIRA
}

Marina Robles Angelini ${ }^{1}$, Angélica Araújo Queiroz $^{2}$
1- $\begin{gathered}\text { Professora Doutora do Curso de Engenharia Agronômica do IFTM, Campus } \\ \text { Uberlândia/MG, marinaangelini@iftm.edu.br }\end{gathered}$
2- Professora Doutora do Curso de Engenharia Agronômica do IFTM, Campus
Uberlândia/MG.
Recebido em: 03/10/2016 - Aprovado em: 21/11/2016 - Publicado em: 05/12/2016
DOI: 10.18677/EnciBio_2016B_010

\section{RESUMO}

Dentre os problemas fitossanitários que ocorrem na cultura da bananeira destaca-se o ataque de pragas, Cosmopolites sordidus considerado a principal praga da cultura. Para seu controle são utilizados vários métodos, sendo os mais comuns o cultural e o químico. Outro método que pode ser utilizado é o uso de variedades resistentes, no entanto, existem poucos relatos sobre a reação de genótipos de bananeira a essa praga. Objetivou-se avaliar a atratividade de quatro variedades de bananeira, Grande Naine, Mysore, Nanicão e Marmelo, em relação à broca-do-rizoma, no município de Uberlândia/MG. Primeiramente avaliou-se a atratividade das diferentes variedades em laboratório, em testes com e sem chance de escolha e posteriormente foram utilizadas, no campo, iscas do tipo telha confeccionadas com um pedaço de pseudocaule para avaliar a atratividade. Nos testes com e sem chance de escolha, foram utilizadas placas de Petri que receberam no seu interior pedaços de pseudocaule das variedades estudadas e em seguida adultos da praga foram liberados, anotando-se o número de insetos em cada material em diferentes tempos de observação. A segunda etapa do experimento foi realizada em área cultivada por plantas com aproximadamente quatro anos de idade, sendo a avaliação dos genótipos realizada através de iscas do tipo telha dos genótipos estudados. A variedade Grande Naine mostra-se menos atrativa aos adultos de $C$. sordidus, quando comparada a Mysore, Nanicão e Marmelo na região de Uberlândia/MG.

PALAVRAS-CHAVE - Broca do rizoma, Musa sp., Resistência de plantas a insetos.

\section{ATTRACTIVENESS OF COSMOPOLITES SORDIDUS IN BANANA GENOTYPES}

\begin{abstract}
Among the plant health problems which occur in banana plant crop highlights the pest attack, Cosmopolites sordidus considered the main pest of the crop. In order to control are used several methods, the most common being the cultural and chemical. Another method that can be used is the resistant varieties; however, there are few reports on the reaction of banana crop genotypes to this pest. This study was carried out in order to evaluate the attractiveness of four varieties of banana plant, "Grande Naine", "Mysore", "Nanicão" and "Marmelo", in relation to the banana root borer (C.
\end{abstract}


sordidus), in Uberlândia / MG. Firstly was evaluated the attractiveness of different varieties in laboratory tests with and without choice and were subsequently used in the field, tile type baits made with a piece of pseudostem to assess the attractiveness. In the tests with and without choice, Petri plates were used which received inside pieces of pseudostem varieties studied and adults of the pest then were released, counting the number of insects in each material at different times of observation. The second stage of the study was carried out in the cultivated area of plants with about four years old, with a genotypes assessment performed by tile type baits of genotypes. The variety "Grande Naine" shown to be less attractive to $C$. sordidus adults compared to "Mysore", "Nanicão" and "Marmelo" in Uberlândia / MG region.

KEYWORDS: Banana root borrer, Musa sp., host plant resistance.

\section{INTRODUÇÃO}

A banana é a fruta mais consumida no Brasil e a segunda no mundo, seguida apenas da laranja. Ocupa a primeira posição no ranking mundial de produção de frutas, com mais de 106 milhões de toneladas, sendo que o Brasil responde por sete milhões de toneladas, com participação de 6,9\% desse total. Dentre os maiores estados produtores da fruta destaca-se São Paulo e Bahia com mais de 30\% da produção nacional na safra 2014/2015 (IBGE, 2016).

Minas Gerais figura como terceiro Estado produtor, contribuindo em média com 11,4 da produção brasileira de bananas (BRASIL, 2016). O Triângulo Mineiro possui cerca de 2.000 ha cultivados com banana, onde foram produzidas 30,4 mil toneladas da fruta (IBGE, 2016), o que confirma a importância da bananicultura para a região, especialmente das cultivares Prata, Maçã e Terra.

As cultivares mais exploradas no Brasil são a Prata, a Pacovan, a Prata-Anã, a Maçã, a Mysore e a Terra, utilizadas para o mercado interno e a Nanica, a Nanicão e Grande Naine, preferidas pelo mercado internacional, sendo que existem outras cultivares até então pouco exploradas, como é o caso dos cultivares BRS Platina, BRS Conquista e FHIA-18 (REETZ et al., 2015).

Embora o Brasil seja um dos maiores produtores mundiais de banana, exporta somente uma pequena parcela de sua produção. Esta baixa representatividade do Brasil está relacionada principalmente à baixa produtividade e ao alto índice de perdas, causadas principalmente por fatores fitossanitários, que comprometem a produção dessa fruta.

Dentre os fatores fitossanitários, destaca-se o ataque de insetos-praga como Cosmopolites sordidus (Germar), conhecido também como "broca-do rizoma" ou moleque-da-bananeira. Esse inseto encontra-se distribuído por todas as regiões do Brasil, sendo uma das pragas mais preocupantes para a cultura. A larva da broca constrói galerias no interior do rizoma, onde são armazenadas reservas para o sustento de todos os outros órgãos da planta. As galerias debilitam as plantas, tornando-as mais sensíveis ao tombamento, sobretudo aquelas que se encontram na fase de frutificação. As bananeiras infectadas apresentam desenvolvimento limitado, diminuem a produtividade e os frutos são curtos e finos (BIOCONTROLE, 2016).

Dentre as medidas de controle para redução populacional da broca-dorizoma, destaca-se o uso de mudas sadias, cultivares resistentes, manejo cultural, iscas atrativas, controle biológico, controle por comportamento e químico (FANCELLI et al., 2015). Entre as variedades de bananeiras cultivadas, não existem espécies consideradas verdadeiramente resistentes, porém, no Brasil, foi constatado que as 
cultivares 'Maçã' e 'Terra' são mais suscetíveis ao ataque de $C$. sordidus, do que as cultivares 'Prata', 'Nanica' e 'Nanicão'(BATISTA FILHO et al. 2002), sendo que esses fatores de resistência e susceptibilidade são variáveis em função do local e as condições de cultivo. Diante do exposto, objetivou-se avaliar o comportamento de genótipos de bananeira em relação à $C$. sordidus, na região de Uberlândia/MG.

\section{MATERIAL E MÉTODOS}

Primeiramente estudou-se a atratividade de adultos de $C$. sordidus por variedades de bananeira, em testes com e sem chance de escolha, em condições de laboratório. Concluída essa etapa foi realizada a atratividade dos genótipos em condições de campo.

Para o estudo da atratividade em testes com e sem chance de escolha foi utilizado delineamento inteiramente casualizado com quatro tratamentos (variedades) e seis repetições, sendo as variedades estudadas Grande Naine, Nanicão, Mysore e Marmelo.

Para o teste com chance de escolha, placas de Petri $(15 \mathrm{~cm}$ de diâmetro) tiveram o fundo revestido com papel filtro umedecido em água destilada. Posteriormente, no interior de cada placa foi colocado um pedaço de pseudocaule de cada material avaliado liberando-se em seguida dois adultos dos insetos por variedade. Para o teste sem chance de escolha, a metodologia utilizada foi similar, no entanto, foram utilizadas placas de Petri menores $(9 \mathrm{~cm}$ de diâmetro), onde colocou-se apenas um segmento de pseudocaule de cada material estudado por placa. Em seguida um adulto de $C$. sordidus foi liberado no interior de cada placa $e$ em seguida foi anotado o número de insetos em cada material estudado em diferentes tempos de observação $(10,15,30,60,120,240,1440$ e 2880 minutos após a liberação), a fim de se determinar a atratividade desses pelas diferentes variedades.

A segunda etapa do experimento foi realizada em uma área cultivada por plantas de bananeiras das variedades Grande Naine, Nanicão, Mysore e Marmelo, com aproximadamente quatro anos de idade, não tratada com inseticidas. A parcela experimental consistiu de quatro linhas de oito plantas de cada genótipo, plantadas no espaçamento $3,0 \times 2,5 \mathrm{~m}$.

A avaliação da atratividade dos genótipos a $C$. sordidus foi realizada com a utilização de cinco iscas do tipo telha de cada genótipo estudado, confeccionadas com um pedaço de pseudocaule de aproximadamente $50 \mathrm{~cm}$ de comprimento, seccionado longitudinalmente ao meio. As iscas dos quatro genótipos estudados foram colocadas, equidistantes, próximas à base das plantas, com a parte seccionada voltada para o solo, previamente limpo.

As avaliações foram realizadas semanalmente, contando-se o número de insetos adultos/isca, durante seis (6) semanas. Após a contagem, os insetos encontrados foram retirados das iscas e liberados no local, sendo as iscas substituídas, após cada avaliação, objetivando manter a atratividade das mesmas.

A significância do efeito dos tratamentos foi estimada por meio do Teste $F$, sendo os níveis do fator qualitativo (genótipo) comparado por meio do Teste de Tukey e os do fator quantitativo (tempos) por meio de análise de regressão. 


\section{RESULTADOS E DISCUSSÃO \\ Atratividade de Cosmopolites sordidus por variedades de bananeira, em testes com e sem chance de escolha.}

Analisando-se a atratividade de $C$. sordidus pelos genótipos estudados, observa-se diferença (Tabela 1) no teste com chance de escolha. Nesse caso, a maior preferência dos adultos da praga foi pelo genótipo Mysore, o qual atraiu ao final das avaliações em média 2,62 insetos. Entre os demais genótipos testados não houve diferença. Considerando-se os diferentes tempos de observação, não houve diferença na atração dos insetos (Tabela 2).

TABELA 1. Número médio de Cosmopolites sordidus atraídos por diferentes genótipos de bananeira, em teste com chance de escolha.
Genótipo
Número médio de insetos atraídos

\begin{tabular}{cc}
\hline Grande Naine & $0,87 \mathrm{~b}$ \\
Nanicão & $1,52 \mathrm{~b}$ \\
Mysore & $2,62 \mathrm{a}$ \\
Marmelo & $0,89 \mathrm{~b}$ \\
\hline (F1= genótipo) & $11.51^{\star *}$ \\
(F2 = tempo) & $2,76^{--}$ \\
(F1xF2) & $0,54^{\text {ns }}$ \\
\hline
\end{tabular}

Médias seguidas pela mesma letra, na coluna, não diferem entre si pelo teste de Tukey a $5 \%$ de probabilidade. ${ }^{* *}$ significativo a $1 \%$ de probabilidade; ${ }^{\text {ns }}$ não significativo; ' ${ }^{-\prime}$ teste $\mathrm{F}$ não se aplica.

TABELA 2. Número médio de Cosmopolites sordidus atraídos pelos genótipos de bananeira em diferentes tempos de observação, em teste com chance de escolha. 2016.

\begin{tabular}{cc}
\hline Tempo de observação & Número médio de insetos atraídos $^{\text {ns }}$ \\
\hline 10 minutos & 0,37 \\
15 minutos & 0,83 \\
30 minutos & 1,54 \\
60 minutos & 1,87 \\
120 minutos & 1,62 \\
240 minutos & 1,91 \\
1440 minutos & 1,79 \\
2880 minutos & 1,87 \\
\hline
\end{tabular}

No teste de atratividade sem chance de escolha, houve diferença entre os genótipos estudados (Tabela 3). Nesse caso, Nanicão e Marmelo mostraram-se como mais atrativos aos adultos de $C$. sordidus quando comparado com Grande Naine. Entre os diferentes tempos de observação, não houve diferença na atração dos insetos (Tabela 4). 
TABELA 3. Número médio de Cosmopolites sordidus atraídos por diferentes genótipos de bananeira, em teste sem chance de escolha.

\begin{tabular}{|c|c|}
\hline Genótipo & Número médio de insetos atraídos \\
\hline Grande Naine & $0,60 \mathrm{c}$ \\
\hline Nanicão & $0,92 \mathrm{a}$ \\
\hline Mysore & $0,71 \mathrm{bc}$ \\
\hline Marmelo & $0,89 \mathrm{ab}$ \\
\hline (F1= genótipo) & $7,07^{\star *}$ \\
\hline$(\mathrm{F} 2=$ tempo $)$ & $2,76^{-}$ \\
\hline$(\mathrm{F} 1 \times \mathrm{F} 2)$ & $0,58^{\mathrm{ns}}$ \\
\hline \multirow{2}{*}{\multicolumn{2}{|c|}{$\begin{array}{l}\text { Médias seguidas pela mesma letra, na coluna, não diferem entre si pelo teste de Tukey a } 5 \% \text { de } \\
\text { probabilidade. }{ }^{*} \text { significativo a } 1 \% \text { de probabilidade; }{ }^{\text {;s }} \text { não significativo; }{ }^{-1} \text { teste } \mathrm{F} \text { não se aplica. } \\
\text { TABELA 4. Número médio de Cosmopolites sordidus atraídos pelos genótipos de } \\
\text { bananeira em diferentes tempos de observação, em teste sem chance } \\
\text { de escolha. }\end{array}$}} \\
\hline & \\
\hline Tempo de observação & Número médio de insetos atraídos ${ }^{\text {ns }}$ \\
\hline 10 minutos & 0,75 \\
\hline 15 minutos & 0,75 \\
\hline 30 minutos & 0,87 \\
\hline 60 minutos & 0,87 \\
\hline 120 minutos & 0,83 \\
\hline 240 minutos & 0,96 \\
\hline 1440 minutos & 0,54 \\
\hline 2880 minutos & 0,67 \\
\hline
\end{tabular}

De acordo com FANCELLI (1999), apesar de todas as variedades de bananeira ser atacadas por $C$. sordidus, o grau de infestação varia de acordo com a variedade utilizada. LARA et al. (2000) ao estudarem a atratividade de variedades de bananeira a esse inseto, observaram que rizomas das variedades Prata e Marmelo e pseudocaules de Marmelo, Nanica e Prata foram menos preferidos para alimentação do inseto, em testes com e sem chance de escolha, sendo dessa maneira, as variedades Prata e Marmelo mais resistentes a esse coleóptero. Esses resultados diferem dos com os observados no presente estudo, onde a variedade Marmelo mostrou-se tão atrativa quanto à variedade Nanicão, considerada por BATISTA FILHO (2002) suscetível a C. sordidus.

Já a variedade Grande Naine mostrou-se como a menos atrativa ao inseto, tanto nos teste com e sem chance de escolha. Esses resultados diferem dos observados por RIBEIRO et al. (2009), que ao avaliarem a infestação da broca do rizoma em em seis cultivares de bananeira, concluiram que Grande Naine foi a mais atrativa quando comparada a Tap Maeo, Caipira e Pacovan.

A comunicação química é a principal forma que os insetos utilizam para localizar a planta hospedeira, sendo guiados principalmente pela detecção de misturas características de voláteis emitidos por elas (WEBSTER et al. 2010). No entanto, essas substâncias apresentam-se em quantidades variáveis, de acordo com a idade das plantas, podendo variar, ainda, segundo a localização, grau, tempo e tipo da injúria, o que pode explicar esses diferentes resultados encontrados. Além dos mencionados acima, outros fatores podem alterar a preferência de espécies de 
plantas hospedeiras pelos insetos fitófagos, sendo eles os ambientais como temperatura, umidade, pluviosidade, evapotranspiração, entre outros.

\section{Atratividade de genótipos de bananeira a Cosmopolites sordidus, em condições de campo.}

Em condições de campo, nas três primeiras avaliações realizadas não se observou diferença em relação ao número médio de insetos atraídos pelos genótipos. No entanto, Mysore apresentou uma tendência em ser mais atrativa a $C$. sordidus. Já na $4^{a}$ e na $5^{\text {a }}$ avaliação realizada, Mysore atraiu maior número de insetos, ou seja, em média 4,8 insetos e 4,4 insetos, respectivamente, diferindo das variedades Grand Naine, Nanicão e Marmelo, as quais foram igualmente atrativas aos adultos de $C$. sordidus. $\mathrm{Na} 6^{\underline{a}}$ avaliação realizada 0 comportamento das variedades foi semelhante a avaliação anterior, no entanto, a Marmelo não diferiu das demais variedades estudadas (Tabela 5).

TABELA 5. Número médio de adultos de Cosmopolites sordidus atraídos por iscas do tipo telha, na cultura da bananeira, em condições de campo.

\begin{tabular}{lcccccc}
\hline & \multicolumn{7}{c}{ Avaliações } \\
\cline { 2 - 7 } Variedades & $\mathbf{1}^{\mathbf{a}}$ & $\mathbf{2}^{\mathbf{a}}$ & $\mathbf{3}^{\mathbf{a}}$ & $\mathbf{4}^{\mathbf{a}}$ & $\mathbf{5}^{\mathbf{a}}$ & $\mathbf{6}^{\mathbf{a}}$ \\
\hline Grand Naine & $0,6 \mathrm{a}$ & $0,0 \mathrm{a}$ & $0,0 \mathrm{a}$ & $0,4 \mathrm{~b}$ & $1,0 \mathrm{~b}$ & $0,4 \mathrm{~b}$ \\
Nanicão & $0,2 \mathrm{a}$ & $1,4 \mathrm{a}$ & $0,8 \mathrm{a}$ & $0,0 \mathrm{~b}$ & $2,0 \mathrm{~b}$ & $0,6 \mathrm{~b}$ \\
Mysore & $1,6 \mathrm{a}$ & $2,8 \mathrm{a}$ & $1,0 \mathrm{a}$ & $4,8 \mathrm{a}$ & $4,4 \mathrm{a}$ & $2,8 \mathrm{a}$ \\
Marmelo & $0,8 \mathrm{a}$ & $2,4 \mathrm{a}$ & $0,2 \mathrm{a}$ & $0,8 \mathrm{~b}$ & $3,4 \mathrm{ab}$ & $1,8 \mathrm{ab}$ \\
\hline CV (\%) & 25,50 & 30,63 & 31,29 & 31,28 & 56,76 & 43,37 \\
\hline
\end{tabular}

Médias seguidas pela mesma letra na coluna não diferem entre si pelo teste de Tukey, a $5 \%$ de probabilidade.

MESQUITA et al. (1985) estudando a susceptibilidade de bananeira a $C$. sordidus, observaram que cultivares do grupo Figo vermelho, Ouro e subgrupo Prata foram menos atrativas do que aquelas do Subgrupo Nanica e Leite, que pertencem ao Subgrupo Cavendish, o mesmo Subgrupo da variedade Grand Naine. O resultado do presente trabalho diverge do trabalho realizado por RIBEIRO et al. (2009) ao concluírem que a cultivar mais suscetível foi a Grand Naine, seguida da Thap Maeo.

Resultados diferentes foram encontrados também por BATISTA FILHO et al., (2002) os quais concluíram que as cultivares mais susceptíveis ao coleóptero $C$. sordidus correspondem à são Nanica, Nanicão, Terra, D’Angola, Figo cinza e Figo vermelho. Já as cultivares Pacovan, Prata, Prata-anã, Maçã e Mysore, são menos susceptíveis.

Em estudos realizados por SALUSTINO et al. (2015), C. sordidus teve uma maior preferência pela variedade Nanica, em relação a Pacovã, sendo que em todas as avaliações para as duas variedades, o pseudocaule mostrou-se mais atrativo que o rizoma. Portanto, isto indica que a cultivar Pacovã foi menos atrativa em relação a ENCICLOPÉDIA BIOSFERA, Centro Científico Conhecer - Goiânia, v.13 n.24; p.125 
Nanica, uma evidencia de que podem sofrer menos ataques destes insetos no seu meio natural.

DANTAS et al. (2011) estudando o comportamento de cultivares de bananeira frente ao ataque de $C$. sordidus, no Vale do Açu/RN, observaram que a variedade Caipira apresentou-se como a mais resistente entre as estudadas, com menos lesões de oviposição do inseto. De maneira geral, tem-se notado que as cultivares Terra, D’angola, Nanica e Nanicão são normalmente mais atacadas que Prata, Prata-Anã, Pacovan e Mysore (MILANEZ, 2010).

Vale destacar que divergências entre resultados encontrados podem ser explicadas facilmente pelos genótipos avaliados, condições experimentais e metodologias empregadas. De acordo com FANCELLI (2016), fatores de resistência e susceptibilidade são bastante variáveis conforme o local e as condições de cultivo.

De acordo com DANTAS et al. (2011) são necessários mais estudos sobre a infestação de $C$. sordidus em bananeira, para que possam ser utilizados como suporte para se entender os mecanismos de resistência da cultura em relação a praga em estudo, já que os mecanismos envolvidos são pouco conhecidos, tanto no que se refere a emissão de voláteis como sua interferência na mobilidade dos insetos e na possível variabilidade intraespecífica de $C$. sordidus.

\section{CONCLUSÃO}

A variedade Grande Naine mostra-se como menos atrativa aos adultos de Cosmopolites sordidus, em testes realizados com e sem chance de escolha em condições de laboratório e em condições de campo, quando comparada as variedades Mysore, Nanicão e Marmelo, o que mostra a resistência do tipo nãopreferência dessa variedade a $C$. sordidus na região de Uberlândia/MG.

\section{REFERÊNCIAS}

BARBOSA, C. A. Manual de Cultivo da bananeira. Viçosa: Ed. do autor, 2009.

BATISTA FILHO, A.; TAKADA, H. M.. ; CARVALHO, A G. Brocas da bananeira. In: Reunião Itinerante de Fitossanidade do Instituto Biológico, 6, 2002, São Bento do Sapucaí-SP. Anais... São Paulo: Arquivos do Instituto Biológico, 2002. v.1. p.1-16.

BIOCONTROLE. Cosmopolites sordidus. Disponível em: http://www.biocontrole.com.br. Acesso em 15/09/2016.

BRASIL, Ministério da agropecuária, pecuária e abastecimento. Projeções do Agronegócio- Brasil 2014/15 a 2024/25. Disponível em http://www.agricultura.gov.br. Acesso em 20 de agosto de 2016.

DANTAS, D. J; MEDEIROS, A. C; NUNES, G. H. S.; MENDONÇA V., MOREIRA, M. A. B. Reação de cultivares de bananeira ao Cosmopolites sordidus no Vale do Açu RN. Revista Verde, v.6, n.3, p. 152 - 155. 2011. Disponível em: http://www.gvaa.com.br/revista/index.php/RVADS/article/view/496/697.

FANCELLI, M.; MILANEZ, J. M.; MESQUITA, A.L.M.; COSTA, A.C.F.; COSTA, J. N.M.; PAVARINI, R.; PAVARINI, G. P. P. Artrópodes: pragas da bananeira e controle. Informe Agropecuário, v.36, n.288, p.7-18, 2015. 
FANCELLI, M. Pragas - Banana, Produção. (Frutas do Brasil, 1). Disponível em: http://www.ceinfo.cnpat.embrapa.br/arquivos/artigo_2903.pdf. Acessado dia 05/01/2016.

IBGE. Levantamento sistemático da produção agrícola. Disponível em: http://www.ibge.gov.br. Acesso em: 20 de agosto de 2016.

LARA, F.M.; SARGO, H.L.B ; CAMPOS, A.R. ; BARBOSA, J.C. Preferência de Cosmopolites sordidus GERM. (Coleoptera: Curculionidae), por genótipos de bananeira, em condições de laboratório. Revista Ecossistema, v. 25, n.1, p.35-38, 2000. ISSN: 0100-4107.

MESQUITA, A. L. M. Avaliação do ataque do Cosmopolites sordidus (Germar, 1824) (Coleoptera: Curculionidae) em rizoma de bananeira. Cruz das Almas: Embrapa - CNPMF, 1985. 2p.

REETZ, E. R.; KIST, B. B.; SANTOS, C. E.; CARVALHO, C.; DRUM, M. Anuário Brasileiro da Fruticultura 2014. Santa Cruz do Sul: Editora Gazeta Santa Cruz, 2015. 104p.

RIBEIRO, G.T.; AZEVEDO, R.L.; PODEROSO, J.C.M.; OLIVEIRA, M.E.C. Infestação do moleque da bananeira em variedades de bananeira, na região de Inhambupe Bahia. Revista Caatinga, v. 22, n.2, p. 5-7, 2009. Disponível em:<http://www.redalyc.org/articulo.oa?id=237117600037 ISSN 0100-316X.

SALUSTINO, A. S; MEDEIROS, M. B.; OLIVEIRA, M. F.; OLIVEIRA, I. S. S. Atratividade olfatométrica de Cosmopolites sordidus Germar, 1824 (Col.: Curculionidae) a dois genótipos de banana Musa sp. XV Encontro Regional de Agroecologia. Cadernos de Agroecologia - ISSN 2236-7934 - Vol 10, № 2 de 2015.

WEBSTER, B.; BRUCE, T.; PICKETT, J.; HARDIE, J. Volatiles functioning as host cues in a blend become nonhost cues when presented alone to the black bean aphid. Animal Behaviour, v. 79, p. 451-457. 2010. DOI:10.1016 